\title{
Estudantes africanas imigrantes no Ceará - análise do processo adaptativo
}

\author{
African immigrant students in Ceará - \\ adaptive process analysis
}

\section{Geraldo Flamarion da Ponte Liberato Filho', Zélia Maria de Sousa Araújo Santos², José Manuel Peixoto Caldas ${ }^{3}$, Maria Helena de Agrela Gonçalves Jardim4, Maria Regina Teixeira Ferreira Capelo ${ }^{5}$, Paula Dayanna Sousa dos Santos ${ }^{6}$}

'Universidade de Fortaleza. Fortaleza, Ceará, Brasil. ORCID: 0000-0001-6648-8991.gflamarion@hotmail.com ${ }^{2}$ Autora para correspondência. Universidade de Fortaleza. Fortaleza, Ceará, Brasil. ORCID: 0000-0002-5824-0723. zeliasantos@unifor.br 3Universidade do Porto. Porto, Portugal. ORCID: 0000-0003-0796-1595.jmpeixotocaldas@gmail.com ${ }^{4}$ Universidade da Madeira. Funchal, Madeira, Portugal. ORCID: 0000-0001-7622-1449. hjardim@uma.pt 5Universidade da Madeira. Funchal, Madeira, Portugal. ORCID: 000-0003-0423-8676. m.regina.capeli@gmail.com ${ }^{6}$ Hospital de Messejana Doutor Carlos Alberto Studart Gomes. Fortaleza, Ceará, Brasil. ORCID: 0000-0001-7653-666x. pauladayanna@hotmail.com

RESUMO | Estudo de caso com o objetivo de analisar o processo adaptativo de estudantes africanas imigrantes no Ceará, realizado na Universidade da Integração Internacional da Lusofonia Afro-Brasileira em Redenção-Ceará-Brasil. Participaram do estudo onze estudantes procedentes de Países Africanos de Língua Oficial Portuguesa (PALOP'S). De acordo com a nacionalidade, incluímos: três de Moçambique; duas de São Tomé e Príncipe; duas de Cabo verde; duas de Angola; e duas de Guiné-Bissau. As estudantes estavam na faixa etária de 22 a 32 anos. Com relação à renda mensal, a individual variava de $R \$ 500,00$ a $R \$ 1.020,00$, e a familiar de $R \$ 500,00$ a $R \$ 1.300,00$. Dentre as estudantes, identificamos fatores de risco para Síndrome Hipertensiva da Gravidez, além da cor negra. Os motivos da migração estavam relacionados com a busca de uma formação acadêmica, conquista de melhores condições de trabalho e de realização profissional e disponibilizar melhorias para os familiares e conhecidos do País de origem mediante o seu regresso. Para sete estudantes houve facilidades, e estas se relacionavam com oportunidade de estudo financiado, liberdade, segurança, melhores condições de vida, e amadurecimento. As dificuldades de adaptação no País eram inerentes ao tipo de alimentação, clima, cultura, racismo/preconceito, custo com moradia, acesso aos serviços de saúde, insegurança e violência. A partir da análise dos resultados consideramos que as estudantes imigrantes africanas enfrentavam dificuldades no processo adaptativo migratório mediadas pela diferença cultural, discriminação, racismo e poder aquisitivo aquém das ofertas locais relacionadas ao atendimento às suas necessidades básicas.
ABSTRACT | Case study with the objective of analyzing the adaptive process of African immigrant students in Ceará, at the University of International Integration of Afro-Brazilian Lusophony in Redenção-Ceará-Brazil. Eleven (11) students from PortugueseSpeaking African Countries (PALOP'S) participated in the study. According to nationality, we include: 03 (three) from Mozambique; 02 (two) of Sao Tome and Principe; 02 (two) Green cable; 02 (two) from Angola; and 02 (two) from Guinea-Bissau. The students were in the age group of 22 to 32 years. In relation to the monthly income, the individual ranged from $R \$ 500.00$ to $R \$ 1,020.00$, and the family from $R \$ 500.00$ to $R \$ 1.300 .00$. Among the students, we identified risk factors for Hypertensive Pregnancy Syndrome in addition to black. The reasons for the migration were related to the search for an academic formation, to obtain better conditions of work and professional achievement and to make improvements to the relatives and acquaintances of the Country of origin upon their return. For seven students there were facilities, and these were related to opportunity for study funded, freedom, security, better living conditions, and maturity. The difficulties of adaptation in the country were inherent to the type of food, climate, culture, racism / prejudice, cost of housing, access to health services, insecurity and violence. From the analysis of the results, we consider that African immigrant students faced difficulties in the adaptive migratory process mediated by cultural difference, discrimination, racism and purchasing power short of local offerings related to meeting their basic needs.

KEYWORDS: Student. Adaptation. Immigration. 


\section{Introdução}

As tendências migratórias constituem uma realidade global incontornável que contrariam o envelhecimento demográfico e atendem às necessidades do mercado de trabalho, contribuições essenciais para o desenvolvimento econômico e sociocultural. A migração é também um desafio, porque novos requisitos surgem, quando a população se torna mais heterogénea, e as sociedades têm de se adaptar a um novo contexto de coexistência. Um dos mais importantes desafios concomitantes à migração é a prestação universal e equitativa de cuidados à saúde. Que estes sejam acessíveis e tenham qualidade para toda a população, independentemente do sexo, etnia ou país de origem, pois é uma questão de direitos humanos, conforme tem sido aludido no Relatório de Desenvolvimento Humano das Nações Unidas, Portugal e Brasil, que têm demonstrado empenho em melhorar a integração dos seus migrantes. Saúde e acesso aos cuidados de saúde são aspectos essenciais da inclusão ou exclusão social dos imigrantes (Ingleby, 2012; Cavalcanti, 2015).

Mulheres migrantes iniciam com maior frequência seu processo de mobilidade antes mesmo ou até durante a idade de procriação, independentemente das motivações pessoais e sociais que levam a deixar o seu país. Estas, uma vez confrontados com os novos contextos societais estão expostas a riscos biológicos, ambientais e psicossociais, acentuando situações de vulnerabilidade social (Fernandes, \& Miguel, 2009; Bunevicius, Kusminskas, Bunevicius, Nadisauskiene, Jureniene, \& Pop, 2009).

O processo do estresse associado ao processo da migração pode ser um desencadeador importante de complicações psicológicas tais como o estresse pós-parto, ansiedade e depressão (Bunevicius et al, 2009; Rumbold, Bailie, Ross, Si, Dowden, Kennedy, Cox, O'Donoghue, Liddle, Kwedza, Thompson, Burke, Brow, Weeramanthri, \& Connors, 2011). Muitos destes fatores de risco são motivados pela falta de apoio social, desenraizamento sociocultural (próprios do processo migratório), que trazem consigo alterações de personalidade. Daí sermos confrontados cotidianamente com situações de partos prematuros, recém-nascidos com baixo peso e maior mortalidade infantil entre a população imigrante (Eastwood, Phung, \& Barnet, 2011). Além disso, os migrantes geram expectativas sobre cuidados de saúde recebidos durante a gravidez geralmente deferindo dos conflitos com os procedimentos normativos do país de acolhimento (Dias, Gama, \& Rocha, 2010; Dias, Severo, \& Barros, 2008).

A questão da vulnerabilidade adquire contornos mais alarmantes quando existem barreiras que impedem o acesso das populações migrantes para o sistema de saúde, tais como aquelas relacionadas às dificuldades econômicas, problemas de linguagem, de mobilidade, bem como status legal, as atitudes do prestador de cuidados de saúde, e as diferenças culturais (Dias et al., 2010).

Os migrantes tendem a ser os grupos populacionais de maior vulnerabilidade, especialmente as mulheres em idade fértil, que mostram regularmente maior mortalidade fetal e neonatal, e mais complicações durante a gravidez e pós-parto (Bunevicius et al., 2009; Rumbold et al., 2011; Almeida, Costa-Santos, Caldas, Dias, \& Ayres-de-Campos, 2016).

O bom estado de saúde deste coletivo é fundamental para o futuro dos países que os recebem. Estudos têm demonstrado que as migrantes apresentam piores indicadores de saúde, nomeadamente durante os períodos de gestação ou puerpério; maiores níveis de mortalidade materna, neonatal e mortalidade infantil, abortos espontâneos, maior incidência de depressão pós-parto; e recém-nascidos com baixo peso; e consequência de baixíssimo seguimento ginecológico e deficiente educação pré-natal (Machado, Santana, Carreiro, Nogueira, Barroso, \& Dias, 2007; Almeida et al., 2016).

A migração acadêmica dos africanos de língua portuguesa que vieram para o Ceará, em busca de formação educacional recorreu a entidades de ensino público existentes, como é o caso da Universidade Estadual do Ceará - UECE, Universidade Federal do Ceará - UFC e a Universidade da Integração Internacional da Lusofonia Afro-Brasileira - UNILAB. Esta última teve sua criação durante a sansão do Projeto de Lei n. ${ }^{0} 12.288$ de 20/06/2010, que criou o Estatuto da Igualdade Racial, e teve por objetivo, promover políticas públicas de igualdade de oportunidades e combate à discriminação (Langa, 2016a; Brasil, 2016).

O binômio saúde versus migração desta população estudantil está regida pelo Decreto n. ${ }^{\circ} 7.948$ de 2013 referente aos Programas de Estudantes-Convênio de Graduação (PEC-G) e Convênio de Pós-graduação $(\mathrm{PEC})$ os quais conferem aos estudantes livre acesso 
aos cuidados universais do uso de serviços públicos de saúde através da Lei n. ${ }^{\circ} 8.080$ de 1990 que regulamenta o Sistema Único de Saúde (SUS) (Ministério das Relações Exteriores, 2015; Brasil, 2016).

De acordo com o Decreto n. ${ }^{\circ} 7.948$ de 12 de março de 2013, no Capítulo V do Artigo $14 .^{\circ}$ referente ao PEC-G, " é garantida ao estudante-convênio assistência médica, odontológica e farmacêutica pelo SUS, nos termos de convênio firmado entre o Ministério das Relações Exteriores e o Ministério da Saúde, sem prejuízo da adesão do estudante a um plano de saúde complementar (Decreto $n^{\circ} 7.948,2013$ ).

Somente os cidadãos dos Países Africanos de Língua Oficial Portuguesa (PALOP'S) - Angola, Cabo-Verde, Guiné-Bissau, Moçambique, São Tomé e Príncipe e Timor-Leste, e não os demais africanos ou até mesmo de outras nacionalidades não poderiam estar inseridos neste processo migratório regulamentado? Langa (2015) justifica esse fato pela proximidade linguística e cultural que num primeiro momento parece ser um fator integrador e de inclusão, e por ser o coletivo migrante mais representativo e numeroso residente no Estado do Ceará.

A grande questão que se coloca desde logo é a de saber como é que este coletivo ascende à rede nacional de saúde existente, sabendo de antemão que a Carta Magna Brasileira, ou seja, a Constituição de 1988 confere a todos os cidadãos, sejam nacionais ou estrangeiros os mesmos direitos no livre acesso ao SUS: equidade, integralidade e universalidade. Importante desde logo relembrar que estre trinômio está fundamentado na gratuidade (Langa, 2016b; Có, 2011).

O SUS criado pela Lei 8080/90, indica princípios normativos - universalidade, integralidade e equidade, que asseguram à população direito de cidadania, isto é, todo ser humano tem direito a um padrão de vida capaz de assegurar a ele e à sua família, saúde e bem-estar, sendo este direito de todos e dever do Estado (Barbiani, Junges, Nora, \& Asquidamini, 2014).

Mediante a problemática citada, questionamos: como está sendo a adaptação de estudantes imigrantes no processo migratório? Diante deste questionamento, optamos por este estudo com o objetivo de analisar o processo adaptativo de estudantes africanas imigrantes no Ceará.

\section{Método}

Estudo de caso realizado na Universidade da Integração Internacional da Lusofonia Afro-Brasileira (UNILAB), em Redenção-CE. A UNILAB está vinculada ao Ministério da Educação (MEC) da República Federativa do Brasil, com sede em Redenção-CE, foi instituída pela Lei n 12.289, de 20 de julho de 2010, e instalada em 25 de maio de 2011.

Participaram do estudo 11 (onze) estudantes procedentes de Países Africanos de Língua Oficial Portuguesa (PALOP'S). De acordo com a nacionalidade, incluímos: 3 (três) de Moçambique; 2 (duas) de São Tomé e Príncipe; 2 (duas) de Cabo verde; 2 (duas) de Angola; e 2 (duas) de Guiné-Bissau. O critério de seleção das entrevistadas foi tão somente o fato de estarem regularmente matriculadas na UNILAB.

Construímos a seguinte questão norteadora: “Como foi ou como está sendo o seu processo de adaptação?

A coleta de dados aconteceu durante os meses de julho a outubro de 2017, por meio de duas técnicas: formulário, cujo instrumento conteve dados sócio demográficos e sanitários, e entrevista semiestruturada com a questão norteadora. Realizamos as entrevistas na Instituição de Ensino Superior (IES) citada, conforme o horário e local combinados previamente com as estudantes. O número de participantes foi determinado pela saturação das informações. As entrevistas foram manuscritas, porque a gravação não foi autorizada pelas participantes.

As informações foram organizadas a partir da análise de conteúdo, seguindo os postulados de Bardin (2014), conforme as etapas para a análise e interpretação dos dados coletados: Pré-análise - efetuamos uma leitura exaustiva das entrevistas; Exploração do material - após a leitura, escolhemos as unidades de registro e seguir identificamos e construiremos as categorias; e Tratamento dos resultados - a partir dos resultados obtidos, procedemos à interpretação, fundamentando na literatura selecionada.

Esta pesquisa foi desenvolvida de acordo com a Resolução n. ${ }^{\circ}$ 466/12 da Comissão Nacional de Ética em Pesquisa (CONEP) (Resolução $n^{\circ} 466,2012$ ), que regulamenta a pesquisa com seres humanos. Às participantes foram assegurados 0 anonimato e o 
direito de retirar o consentimento no momento que desejarem. Os dados foram coletados após a assinatura do Termo de Consentimento Livre e Esclarecido e da emissão do parecer favorável da Comissão de Ética da Universidade de Fortaleza - UNIFOR, sob o número 56818116.5.0000.5052. As estudantes foram identificadas pela letra I, seguida da nacionalidade e do número de participantes (I01 nacionalidade a I11 nacionalidade), a fim de garantir o anonimato nas citações de seus discursos.

\section{Resultados}

\section{Caracterização das estudantes}

As mulheres estavam na faixa etária de 22 a 32 anos; quatro eram solteiras, seis conviviam com o companheiro em união estável, e uma estava separada; quanto à prática religiosa, cinco informaram o catolicismo, quatro a religião evangélica, e uma o candomblé; e procedência, sete residiam em Redenção-CE, e quatro em Acarape-CE. Com relação à renda mensal, a individual variava de $R \$ 500,00$ a $R \$ 1.020,00$, e a familiar de $R \$ 500,00$ a $R \$ 1.300,00$. Ressaltamos que seis coabitavam com companheiro, filho (s) e amigas, e as demais com filho (s) e amigas.

Relativamente aos dados sanitários, identificamos fatores de risco para Síndrome Hipertensiva da Gravidez (SHG) além da cor negra, que se seguem: dez primigestas; duas hipertensas; duas com história familiar de SHG; duas faziam uso de bebidas alcoólicas; uma com sobrepeso; uma multípara; uma informou pressão arterial (PA) elevada na gravidez anterior; uma diabética; e uma com gravidez gemelar. As mulheres estavam com esquema vacinal atualizado, e duas eram expostas ao tabagismo do companheiro.

As categorias de análise emergentes da análise das entrevistas sobre a adaptação ao processo migratório foram: tempo de permanência das participantes no Brasil; motivos da migração; e processo Adaptativo no Brasil.

\section{Tempo de permanência das participantes}

O tempo de permanência das mulheres no Brasil, e precisamente no Ceará variava de um a três anos. Os fluxos migratórios são hoje mais numerosos, mais rápidos, mais diversificados e complexos do que no passado, atingindo todos os continentes, gêneros, classes sociais, gerações e os vários domínios da vida pública. As questões da saúde, da qualidade de vida, dos direitos humanos e da cidadania das populações migrantes estão no centro das preocupações de muitos países da União Europeia, mas também de muitos outros Latino-americanos como o Brasil, dos profissionais e dos investigadores. O acesso à saúde é um direito fundamental indispensável para o exercício dos outros direitos humanos. Todavia, as múltiplas e complexas relações e variáveis individuais e coletivas, nomeadamente psicológicas, sociais, culturais, ambientais e políticas, implicadas nas questões da saúde e da doença, na acessibilidade aos serviços de saúde e, ainda, no processo migratório e de aculturação.

\section{Motivos da migração}

De acordo com os relatos das estudantes, os motivos da migração estavam relacionados com a busca de uma formação acadêmica, conquista de melhores condições de trabalho e de realização profissional (segurança/estabilidade), e disponibilizar melhorias para os familiares e conhecidos do País de origem mediante o seu regresso:

[...] buscar melhorias nos estudos e formação profissional a partir de uma graduação [...] (I CV4)

[...] buscar por melhores condições de educação e uma formação profissional internacional [...] (I GB8)

[...] em busca de melhores condições de trabalho e segurança (realização profissional) [...] (I M6)

[...] ganhar conhecimentos para voltar ao meu Pais e ajudar os que necessitam [...] (I CV3)

[...] iniciar os meus estudos e buscar melhorias para o meu povo quando eu voltar [...] (I GB9)

\section{Processo Adaptativo no Brasil}

As estudantes declararam facilidades e dificuldades durante o processo adaptativo. Para sete estudantes houve facilidades, e estas se relacionavam com oportunidade de estudo financiado, liberdade, segurança, melhores condições de vida, e amadurecimento:

\section{[...] a bolsa é pouca, mas já facilita os meus estudos [...]}


[...] liberdade da família, mais oportunidades para os estudos [...] (I CV3)

[...] segurança, melhores condições de vida, aprimoramento dos estudos, amadurecimento [...]

[...] agora eu sou livre, porque eu era muito presa pelos meus pais no Guiné [...] (I GB8)

As dificuldades foram declaradas por todas as estudantes e eram inerentes ao tipo de alimentação, clima, cultura, racismo/preconceito, custo com moradia, acesso aos serviços de saúde, insegurança e violência:

[...] o clima é muito quente e seco, a alimentação é muito industrializada, se você quer comer bem terá que gastar muito. Devido a cultura ou a falta de cultura melhor e mais evoluída e aberta, a população local

é muito fechada com todos nós, são abusivos, nos cobrando alugueis caros onde a estrutura é muito ruim. Além de algumas vezes nos olharem e nos tratarem como indiferença [...] (I A1)

[...] alimentação, moradia, saúde, racismo, falta de financiamentos estudantis, Insegurança. Somos mais assaltadas porque somos negras e migrantes, então eles não se preocupam, se fossem com os brancos locais já teriam resolvido. Fingem que não nos escutam. Acham que não temos direitos [...] (I CV3)

[...] insegurança, moradia, alimentação, custos, racismo. A população local nos trata como se fossemos os escravos e acham que viemos roubar os empregos dos seus filhos e ainda nos cobram muito caro pelos alugueis. Achando que não sabemos os reais valores das coisas [...] (I CV4)

[...] preço moradia muito alto, preconceito, alimentação diferente e cara. Tudo caro e burocrático. Moradia cara, preconceito. Preço alto, para pouco conforto e estrutura ofertada. População local e os profissionais de saúde nos olham diferentes [...] (I M6)

[...] alimentação, clima quente, violência, dificuldade nas consultas, demora em realizar exames e receber.

Todos estes fatores são dificuldades para nossa adaptação. Sem falar que somos descriminados, pois o comportamento dos moradores é diferente com os negros [...] (I GB8)
[...] muita violência, preconceito alimentação diferente, comidas boas e saldáveis é caro no Brasil. Valor da moradia é muito caro e as condições são péssimas [...]

(I STP11)

O processo migratório alenta sonhos, proporciona alegrias, mas em contrapartida oferece inúmeras dificuldades às populações que ousam partir para outros mundos. Sublinha-se a necessidade de serem criadas estratégias e mecanismos de acolhimento para a integração destas populações conforme pressupostos plasmados na Constituição brasileira, uma vez que estas, com o seu esforço e trabalho, poderão cooperar no enriquecimento, bem-estar geral e progresso do país.

\section{Discussão}

Os estudantes africanos integrados às universidades federal e estadual - que constituem, de fato, a minoria - sobrevivem das bolsas do PEC-G e de outros convênios firmados entre o Brasil e seus países de origem. Já o segmento maior, que estuda em faculdades particulares, recebe dinheiro das famílias para pagar mensalidades e manter-se na faculdade, complementando a sua renda por meio de trabalhos considerados irregulares - em lojas e mercadinhos, salões de beleza, oficinas, fábricas e construções, restaurantes ou mesmo, nos estacionamentos de grandes shoppings centers e supermercados, ou, ainda, em "casas de família" como babás - para assim, garantir a sobrevivência e a própria locomoção na cidade (Langa, 2016b).

Os determinantes sociais demonstram que são fortes condicionantes da qualidade de vida em geral e dos imigrantes em particular. Uma baixa qualidade alimentar quase sempre motivada por dificuldades econômicas, propiciadoras de alterações metabólicas, déficit seguimento médico fazem deste coletivo um grupo de risco sanitário importante. Nos últimos anos, são inúmeros os casos de tuberculose entre população imigrante (Almeida et al, 2016).

As estudantes referiram tempo de permanência variável entre um e três anos e colocaram em evidência uma multiplicidade de problemáticas psicológicas, 
sociais, culturais, ambientais, políticas, com implicações na área da saúde, na acessibilidade aos serviços relacionadas com o processo migratório e a própria aculturação. Estas variáveis podem originar isolamento, estresse, ansiedade, depressão, conflito, exclusão e doença, elementos que poderão afetar não apenas a saúde mental e física, o bem-estar e a qualidade de vida dos homens e das mulheres migrantes, como dificultar, igualmente, o seu acesso aos cuidados de saúde e à prevenção, a reivindicação dos seus direitos e o exercício de cidadania (Almeida et al., 2016).

Por sua vez, formação acadêmica, obter melhores condições de trabalho e de realização profissional, designadamente, segurança e estabilidade, e conceder benefícios aos familiares e conhecidos do País de origem mediante o seu regresso foram os motivos apontados para a migração. A oportunidade de estudar num país estrangeiro como o Brasil, onde existe uma língua "teoricamente" comum é vista pela maioria dos imigrantes africanos como um meio para a melhoria de vida no seu País de origem (Langa, 2016a). As emigrações africanas na era da globalização (Malomalo, B., \& Kabubnda Badi, 2015) representam para o continente africano uma oportunidade académico-científica necessária para o desenvolvimento socioeconómico destes países em vias de desenvolvimento.

Foram identificadas facilidades e dificuldades durante o processo adaptativo no testemunho das participantes. Também para Langa (2016a), o "novo mundo" é uma porta de oportunidades para vivências de novas liberdades: políticas, sexuais e sócio afetivas.

A migração luso-africana por motivos académicos estreou-se em 2010 com a criação da Universidade Federal da Integração Luso-Afro-Brasileira (UNILAB) (Langa, 2016a). Assim, na última década do Século $X X$, inicia-se a presença de um novo fluxo de população africana no Estado do Ceará, na sua maioria estudantes, de PALOP'S para integrar-se na Universidade Federal do Ceará (UFC), por meio do Programa de Estudos Convênio - de Graduação (PEC-G) e PósGraduação (PEC-PG) sendo o primeiro grupo oriundo de Angola (Oliveira et al., 2017). As dificuldades foram declaradas por todas as estudantes e eram inerentes ao tipo de alimentação, clima, cultura, custo com moradia, acesso aos serviços de saúde, insegurança, violência e o preconceito racial conducente à discriminação.
Um dos grandes temas que aflorou na análise de conteúdo foi a questão racial, o preconceito e a discriminação a que são submetidos os africanos. Para muitos é a primeira experiência de vida com que se deparam ao chegar a terras cearenses (Langa, 2016b).

Contudo, Carvalho (2012) refere que se trata do longo caminho da cidadania no Brasil. O País da diversidade racial, mas de política mono racial, da invisibilidade do negro e do ostracismo a que são vetados. As situações de preconceito e discriminação racial que permeiam a sociedade brasileira, inclusive a cearense, tem adentrando às IES públicas e particulares, verificando-se diversos casos de racismo sofridos contra estudantes africanos por parte de professores, colegas e corpo técnico administrativo. Entretanto, mesmo denunciando tais situações de racismo e abrindo Boletins de Ocorrência (BO) nas delegacias da cidade, tais situações continuam sucedendo e a aumentar. Ainda assim, diante de dificuldades nas diversas esferas da vida - seja a vida pessoal, estudantil, profissional, no acesso a serviços públicos e privados essenciais como saúde, bancos, Polícia Federal e suas inserções precárias no mercado de emprego e do trabalho - estes sujeitos diaspóricos têm perseverado na luta cotidiana, acreditando que suas condições de inserção na sociedade fortalezense melhorem e sejam aceitos, reconhecidos e integrados como cidadãos.

De acordo com a Constituição Federal do Brasil, o acesso ao Sistema de Saúde de imigrantes deve ser equivalente ao de nativos. Entretanto, aponta Challinor (2012), é preciso reconhecer as diferenças, sob o risco de "diluição" dos imigrantes em nome do tratamento democrático comum. Desse modo, é um desafio aos trabalhadores em saúde o encontro entre diferentes culturas, acentuado no caso do usuário imigrante, valorizando suas crenças e modos de vida, sem impor o poder de cultura dominante, sem vê-lo como "exótico" ou inferior.

De acordo com Ponte Filho, et al (2018), os estudantes africanos ao chegarem ao Brasil, enfrentam desafios cotidianos, particularmente, dificuldades econômicas de sobrevivência, considerando o elevado custo de vida nesta metrópole, em relação às suas possibilidades financeiras, bem como a falta do conhecimento da população acerca da história e da cultura da população negra oriunda da África, gerando o preconceito e a descriminação. 
A organização do processo de trabalho deve ser capaz de promover encontros multiculturais produtores de saúde entre trabalhadores e usuários. Um serviço sobrecarregado de trabalho não é capaz de obter avanços sem uma política de apoio e trabalho conjunto (Challinor, 2012).

Em relação à comunidade PALOP acreditamos que o fato de falarem a língua portuguesa poderá ajudar num primeiro momento, mas percebemos pelas primeiras auscultações que não é bem assim. Muitos destes imigrantes, principalmente os que veem da Guiné-Bissau e Timor-Leste referem que não os entendem, e têm muitos obstáculos linguísticos com os autóctones. Os determinantes sociais da utilização dos serviços de saúde podem ser descritos como aqueles fatores relacionados às: necessidade de saúde; características demográficas; disponibilidade de profissionais de saúde; características de organização dos serviços de saúde; e ao mecanismo de financiamento; é observado menor acesso e consequente menor utilização de serviços de saúde nas populações rurais, pois além da menor disponibilidade de serviços, grandes distâncias a serem percorridas, dificuldades de transporte e baixa renda são fatores que, associados, reduzem a utilização de serviços de saúde nestes ambientes, além da falta de acolhimento nos serviços (Moreira, Moraes, \& Luiz, 2011), 2011; Caldas, \& Santos, 2017).

\section{Considerações finais}

A partir da análise dos resultados consideramos que as estudantes imigrantes africanas enfrentaram dificuldades quanto ao processo adaptativo migratório mediadas pela diferença cultural, discriminação, racismo e poder aquisitivo aquém das ofertas locais relacionadas ao atendimento às suas necessidades básicas.

O fenômeno migratório apresenta crescente feminização, no qual observamos no estudo em pauta algumas estudantes em processo adaptativo já adentram a maternidade, que por si só, remete uma série mudanças na vida destas que contam para a sua sobrevivência o auxílio financeiro dispensado pelo Governo Federal. Mediante esta condição, estas podem tornar-se fragilizadas e expostas aos riscos biológicos e psicossociais. Haja vista que geralmente o imigrante transporta diversos perfis epidemiológicos encubados e multifacetários por meio das suas diver- sas crenças e práticas culturais, as quais inclui o envolvimento do binômio saúde e doença.

As limitações do estudo se limitaram à negativa das estudantes quanto a gravação das entrevistas, e a dificuldade em abordá-las, bem como, entrevistá-las em decorrência do tempo limitado que elas disponham. Pois, estas estudantes ocupavam o seu tempo entre as aulas na IES, os afazeres domésticos, e outras nos cuidados com o filho. Possivelmente, outra dificuldade na abordagem destas estudantes, esteja relacionada ao fato de se sentirem discriminadas, vítimas de racismo pelos nativos (conforme relatos), aspectos que tenham influenciado na aproximação com o pesquisador.

\section{Agradecimentos}

Este artigo é resultado de pesquisa aprovada para apresentação no $7^{\circ}$ Congresso Ibero-Americano em Investigação Qualitativa - CIAIQ, realizado de 10 a 13 de julho de 2018 em Fortaleza, Ceará, Brasil.

\section{Contribuições dos autores}

Liberato Filho, G. F. P. participou da concepção, delineamento, construção dos capítulos de literatura, busca e análise estatística dos dados da pesquisa, interpretação e discussão dos resultados e redação do artigo científico. Santos, Z. M. A. S. participou do delineamento, construção da literatura do artigo, análise estatística dos dados da pesquisa, interpretação e discussão dos resultados e redação do artigo científico. Caldas JMP participou da concepção, delineamento e redação do artigo científico. Jardim, M. H. A. G., Capelo, M. R. T. F. e Santos, P. D. S. participaram da redação do artigo científico.

\section{Conflitos de interesses}

Nenhum conflito financeiro, legal ou político envolvendo terceiros (governo, empresas e fundações privadas, etc.) foi declarado para nenhum aspecto do trabalho submetido (incluindo mas não limitando-se a subvenções e financiamentos, participação em conselho consultivo, desenho de estudo, preparação de manuscrito, análise estatística, etc.).

\section{Referências}

Almeida, L. M., Costa-Santos, C., Caldas, J. P., Dias, S., \& Ayres-deCampos, D. (2016). The impact of migration on womens mental health in the postpartum period. Revista de Saúde Pública, 50, 35. Recuperado de http://www.scielo.br/pdf/ rsp/v50/0034-8910-rsp-S1518-87872016050005617.pdf. doi: 10.1590/S1518-8787.2016050005617 
Barbiani, R., Junges, J. R., Nora, C. R. D., \& Asquidamini, F. (2014). A produção científica sobre acesso no âmbito do Sistema Único de Saúde do Brasil: avanços, limites e desafios. Saúde e Sociedade, 23(3), 855-868. Recuperado de http://www.scielo.br/pdf/sausoc/v23n3/01041290-sausoc-23-3-0855.pdf. doi: 10.1590/50104$\underline{12902014000300010}$

Bardin, L. (2014). Análise de conteúdo. Lisboa: Edições 70.

Bogdan, R. C., \& Biklen, S. K. (1994). Investigação Qualitativa em Educação. Porto: Porto Editora.

Bunevicius, R., Kusminskas, L., Bunevicius, A., Nadisauskiene R., Jureniene, K., \& Pop, V. J. (2009). Psychosocial risk factors for depression during pregnancy. Acta Obstetricia et Gynecologica, 88(5), 599-605. Recuperado https://www.ncbi.nlm.nih.gov/pubmed/19308810. doi: $\underline{10.1080 / 00016340902846049}$

Caldas, J. M. P., \& Santos, Z. M. S. A. (2017). Sobre o direito universal da assistência à saúde. Revista da Rede de Enfermagem do Nordeste, 18(4), 428. Recuperado de http:// periodicos.ufc.br/rene/article/view/20194/30773. doi: 10.15253/2175-6783.2017000400001

Carvalho, J. M. (2012). Cidadania no Brasil: o longo caminho. Rio de Janeiro: Civilização Brasileira.

Cavalcanti, L. (2015). Imigração e mercado de trabalho no Brasil: características e tendências. Cadernos Obmigra - Revista Migrações Intrenacionais, 1, 35-47. Recuperado de https:// notassobremigracion.files.wordpress.com/2015/04/brasilinmig-lab-2015.pdf

Có, J. P. P. (2011). Filhos da Independência: etnografando os estudantes bissau-guineense do PEC-G em Fortaleza-CE e Natal-RN (Dissertação de mestrado). Universidade Federal do Rio Grande do Norte, Natal, RN, Brasil. Recuperado de https://repositorio.ufrn.br/jspui/handle/123456789/12270

Challinor, E. P. (2012). Cidadania médica, culturas e poder nos cuidados perinatais e pediátricos de imigrantes. Saúde e Sociedade, 21(1), 76-88. Recuperado de http://www. scielo.br/pdf/sausoc/v21n1/09.pdf. doi: 10.1590/S010412902012000100009

Decreto $n^{\circ} 7.948$, de 12 de março de 2013. Dispõe sobre o Programa de Estudantes-Convênio de Graduação - PEC-G. Recuperado de http://www.planalto.gov.br/ccivil_03/_ ato2011-2014/2013/decreto/d7948.htm

Dias, S., Gama, A., \& Rocha, C. (2010). Immigrant women's perceptions and experiences of health care services: insights from a focus group study. Journal of Public Health, 18(5), 489-96. Recuperado de https://link.springer.com/ article/10.1007\%2Fs10389-010-0326-x. doi: 10.1007/ s10389-010-0326-X
Dias, S. F., Severo, M., \& Barros, H. (2008). Determinants of health care utilization by immigrants in Portugal. BMC Health Services Res, 8, 207. Recuperado de https://bmchealthservres.biomedcentral.com/ articles/10.1186/1472-6963-8-207. doi: 10.1186/14726963-8-207

Eastwood, J. G., Phung, H., \& Barnett, B. (2011). Postnatal depression and socio-demographic risk: Factors associated with Edinburgh Depression Scale scores in a metropolitan area of New South Wales, Australia. Australian and New Zealand Journal of Psychiaty, 45(12), 1040-1046. Recuperado de https://www.ncbi.nlm.nih.gov/ pubmed/22017687. doi: 10.3109/00048674.2011.619160

Fernandes, A., \& Miguel, J. P. (2009). Health and migration in the European Union: better health for all in an inclusive society. Lisboa: Instituto Nacional de Saúde Doutor Ricardo Jorge.

Ingleby, D. (2012). Ethnicity, Migration and the 'Social Determinantes of Health' Agenda. Psychosocial Intervention, 21(3), 331-341. Recuperado de https://www. sciencedirect.com/science/article/pii/S113205591270087X. doi: 10.5093/in2012a29

Langa, E. N. B. (2016a). Diáspora africana no Ceará no século XXI: Ressignificações identitárias de estudantes imigrantes (Tese de Doutorado). Universidade Federal do Ceará, Fortaleza, CE, Brasil. Recuperado de http://www.repositorio.ufc.br/ handle/riufc/21801

Langa, E. N. B. (2015). Diáspora Africana no Ceará no Século XXI: ressignificações identitárias e as interseccionalidades de raça, gênero, sexualidade e classe no contexto da migração estudantil internacional. In J. D. Fonseca, \& M. K. Badi (Orgs.) Diáspora Africana e migração na era da globalização: experiências de refúgio, estudo, trabalho (pp. 161-186). Curitiba: Editora CRV.

Langa, E. N. B. (2016b). O lugar das mulheres e a questão dos direitos humanos: um olhar sobre experiências, dramas e interseccionalidades de mulheres africanas na cidade de Fortaleza-CE. In M. Rocha (Org.). Direitos Humanos, Sociedade e Política (Coleção Outros Olhares, pp.129-152). Fortaleza: Expressão Gráfica e Editora.

Ministério das Relações Exteriores (2010). Divisão de Temas Educacionais. Programa PEC-G. Recuperado de http://www. dce.mre.gov.br/PEC/PECG.php.

Ministério das Relações Exteriores (2015). Divisão de temas Educacionais. PEC-G: histórico do programa. Brasília. Recuperado de http://www.dce.mre.gov.br/PEC/G/ historico.php 
Ponte Filho, G. F. L., Santos, Z. M. S. A., Caldas, J. M. P., Santos, P. D. S., Paiva, M. C., Palácio, J. S. F., ... Aguiar, A. C. T. (2018, julho). Processo adaptativo de estudantes imigrantes no Estado do Ceará. Atas do Congresso Ibero-Americano em Investigação Qualitativa em Saúde, Fortaleza, CE, Brasil, 7. Recuperado de https://proceedings.ciaiq.org/index.php/ ciaiq2018/article/view/1822

Resolução n. ${ }^{\circ} 466$, de 12 de dezembro de 2012. Aprova diretrizes e normas regulamentadoras de pesquisas envolvendo seres humanos. Recuperado de https://conselho.saude.gov.br/ resolucoes/2012/Reso466.pdf

Machado, M.C., Santana, P., Carreiro, H., Nogueira, H., Barroso, R., \& Dias, A. (2007). Cuidados de saúde materna e infantil a uma população de imigrantes. Migrações, 1 , 103-127. Recuperado de https://www.om.acm.gov.pt/ documents/58428/183863/migracoes1_art5.pdf

Malomalo, B., \& Badi, M. K. (2015). O direito de migrar e o dever de compreender as migrações africanas na era da globalização. In J. D. Fonseca, \& M. K. Badi (Orgs.) Diáspora Africana e migração na era da g/obalização: experiências de refúgio, estudo, trabalho (pp. 9-16). Curitiba: Editora CRV.

Moreira, J. P. L., Moraes, J. R., \& Luiz, R. R. (2011). Utilização de consulta médica e hipertensão arterial sistemática nas áreas urbanas e rurais do Brasil, segundo dados da PNAD 2008. Ciência e Saúde Coletiva, 16( 9), 3781-3793. Recuperado de http://www.scielo.br/pdf/csc/v16n9/ a14v16n9.pdf. doi: 10.1590/S1413-81232011001000014

Oliveira, E. N., Neto, F, \& Lima, G. F. (2017). Violence In Immigrants: Effects On Health, Perception Of Discrimination And Loneliness. International Archives of Medicine, 10(226). Recuperado de http://imedicalsociety.org/ojs/index.php/ iam/article/view/2597. doi: 10.3823/2496

Rumbold, A. R., Bailie, R. S., Si, D., Dowden, M. C., Kennedy, C. M., Cox, R. J., ... Connors, C. M. (2011). Delivery of maternal health care in Indigenous primary care services: Baseline data for an ongoing quality improvement initiative. BMC Pregnancy \& Childbirth, 11-16. Recuperado de https://bmcpregnancychildbirth.biomedcentral.com/ articles/10.1186/1471-2393-11-16. doi: 10.1186/14712393-11-16 